\title{
Binding Study of Cis-Atovaquone with Cytochrome bc1 of Yeast
}

\author{
Srijita Basumallick ${ }^{1,2}$, T. N. Guru Row ${ }^{1}$ \\ ${ }^{1}$ Solid State Chemistry and Structural Unit, Indian Institute of Science, Bangalore, India \\ ${ }^{2}$ Department of Chemistry, National Institute of Technology, Agartala, India \\ Email:srijitabasumallick@gmail.com,ssctng@sscu.iisc.ernet.in
}

Received 24 September 2015; accepted 8 December 2015; published 11 December 2015

Copyright (C) 2015 by authors and Scientific Research Publishing Inc.

This work is licensed under the Creative Commons Attribution International License (CC BY). http://creativecommons.org/licenses/by/4.0/

(c) (i) Open Access

\begin{abstract}
Tans-Atovaquone is widely used as an effective drug to treat uncomplicated malaria. But its cisisomer is not a drug. In the present study, we report energy minimized binding pattern of transAtovaquone and its cis-isomer with cytochrome bc1 (cytbc1) of yeast. The new feature of this molecular docking computation is that structural parameters of the drug molecules have been determined from their crystal structures. The energy minimized structures of protein-drug complexes show that $\mathrm{H}$-bond distant between His-181 of cytochrome bc1 and $\mathrm{C}=0$ of Atovaquone for trans-Atovaquone is $2.85 \AA$ and $5.3 \AA$ with the cis-isomer. The role of this H-bonding interaction in dictating drug potency is in conformity with proton-coupled electron transport mechanism of drug action.
\end{abstract}

\section{Keywords}

\section{Anti-Malarial Drug, Crystal Structures, Atovaquone, Docking-Studies}

\section{Introduction}

According to the recent report on malaria [1], the death of thousands of children is alarming. It is known that malaria is caused mainly by Plasmodium vivax, Plasmodium falciparum and Plasmodium malariae, transmitted through female Anopheles mosquito [2].

Historical developments [3]-[5] in the treatment of malaria started from extraction of quinine from the bark of cinchona in the year 1820.

Derivatives of Quinine like Amodiaquine, Mefloquine, Primaquine [5] are still used in the prevention and treatment of malaria. This class of drug acts [6]-[8] on the food vacuoles of the parasites. Parasites obtain amino acids for protein translation and formation of their cell membrane from digestion of hemoglobin in the host. The 
undigested free toxic heme groups, form reactive oxygen species. The natural process of removing this toxic heme group from the parasitic cell is by the process of bio crystallization of heme to haemozoin [9]. The chloro-quinine inhibits this process of removal of toxic heme groups from parasitic cells and thus kills parasites.

Artemisinin [10] is a redox drug. In metabolic processes, reactive oxygen intermediates are produced as redox wastes. Those can damage the cell components. However, cells have their own mechanism of preventing the formation of redox wastes [11]. The artemisinin-type drug accelerates the formation of these redox wastes in parasites causing parasitic cell death.

Sulfa drugs inhibit activities of two enzymes needed for the folate synthesis [12]; the parasites grow rapidly using nucleic acids synthesized by protein folate [13]. Thus, these drugs suppress parasitic growth.

Atovaquone [14] (Figure 1), is a modern generation drug that acts as the ubiquone (Figure 2) inhibitor in the mechanism of the mitochondrial respiration [15] of the parasitic cells.

The mitochondrial electron transfer (Figure 3) occurs through several consecutive redox reactions as schematically shown below.<smiles>O=C1C=C(C2CCCCC2)C(=O)c2ccccc21</smiles>

Figure 1. The structure of Atovaquone.

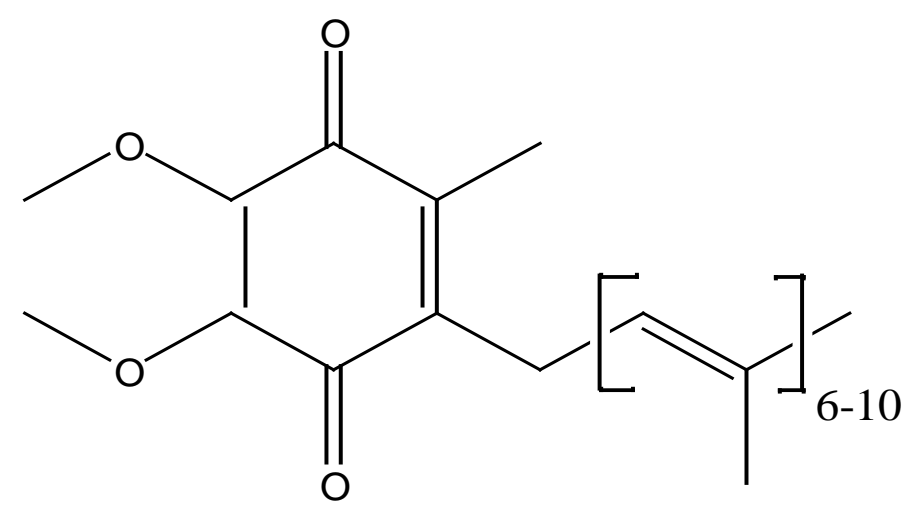

Figure 2. Structure of ubiquinone.

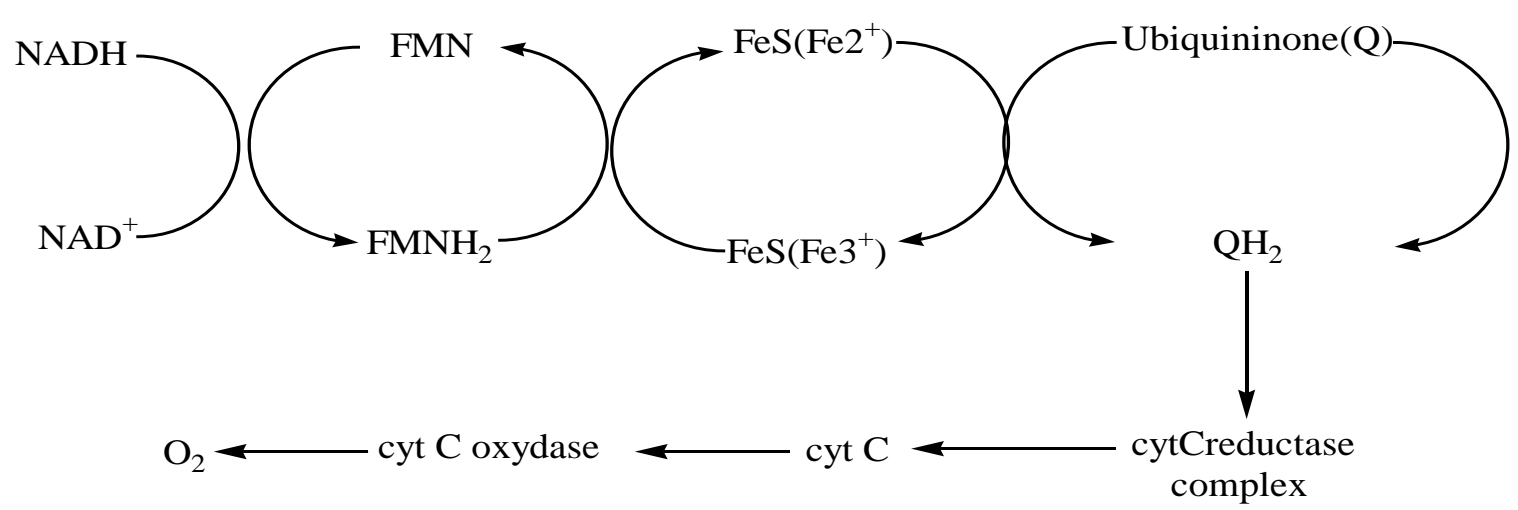

Figure 3. Cytochromebc1 electron transfer path. 
The quinine group of ubiquone gets reduced to the quinol and helps in transferring the electron through an oxidation-reduction cycle [16] [17]. Atovaquone too has a quinine group and thus, it can mimic ubiquone and binds selectively to the $Q_{0}$ site of parasitic mitochondria thereby block the parasitic mitochondrial respiration [14].

In the present study, we report binding characteristics of trans- and cis-isomers of Atovaquone with cytochrome bc1 of yeast using docking technique in order to address the basic question, why cis-Atovaquone is not an anti-malarial drug? We have carried out the computation with cytochrome bc1 of yeast as experimental crystallographic data of Atovaquone-bound mitochondrial cyt bc1 of yeast are available in a very recent literature [18] report.

Recently, Hunte et al. reported [18] [19] crystal structure analysis of Atovaquone-bound cytochrome be1 complex of yeast and discussed [19] [20] elaborately different binding features of the drug molecule but it was limited to trans-Atovaquone only. Despite several studies, the molecular mechanism of ubiquinol oxidation is not yet clearly known. The mechanism of bifurcated electron transfer paths involves transport of one electron via the $2 \mathrm{Fe}-2 \mathrm{~S}$ path of Rieske protein, and the other electron is transported via b-type heme. The-NH centre of His-181 of Rieske protein plays an important role [18] in such electron transport process. Thus, if this centre is blocked by an H-bond with the drug molecule, it will interrupt the normal mitochondrial electron transport process.

Unlike our previous model [21], we have excluded here $\mathrm{H}_{2} \mathrm{O}$ assisted $\mathrm{H}$-bonding interaction between Glu272 and Atovaquone, as this interaction is absent in the experimentally determined crystal structure [18] of Atovaquone bound cytbc1.

\section{Computational Methods}

In the present computation method, simulated annealing [21] [22] process of docking was adopted. The drug molecule (ligand) was considered flexible in terms of its translational orientation and conformational changes. The Auto Dock software (3.0) calculated the local energy minima for different configurations of the drug molecule at the binding site of cytochrome bc1 to obtain the global minimum structure. Monte Carlo simulated annealing technique [23] was used for configuration exploration. The energy calculation was done by the grid based affinity potential [24]. Auto Dock version 3.0 employed local search method based on the optimization of algorithm of Solis and Wets (SW) [25] and global search method by genetic algorithm (GA).

In this approach, the input parameters like atomic coordinates for the cytochrome bc1 was taken from protein data bank and represented "extended" atom representation where the amino, hydroxyl, methyl groups are treated as the single entity. Thus, a single extended amino group replaces four real atoms to reduce the time of computation. The calculation of the energy at different points was obtained by the grid calculation with the drug molecule kept at different grid points. The energy at a grid point was calculated on the basis of affinity potential using the classical Lenard Jones potential and electrostatic potential calculated by considering the atoms as point charges of +1 using a Poison-Boltzmann finite difference method [26]. After calculating the energy at the eight grid points surrounding any atom, an average energy was obtained. The total energy was calculated considering three main interactions 1) Lenard Jones interaction; 2) Electro static interaction; 3) The hydrogen bonding interaction. It may be mentioned here that the novelty of this computation is that coordinates of the drug molecules were obtained from experimentally determined crystal structures of these molecules. The cytochrome bc1 protein orthogonal crystal coordinates were obtained by deleting Stigmatellin coordinates from reported Stigmatellin + cytochrome bc1 protein crystal (PDB code-1EZV) coordinates. The combination of the orthogonal coordinates of the Atovaquone with those of the protein [27] was done by the software win Coot version 0.3.3 [27]. The PyMOL windows version 0.99 was used for viewing and drawing the energy minimized structure of Atovaquone bound cytochrome bc1 complexes.

\section{Result and Discussion}

\subsection{Binding Features of Trans-Atovaquone}

Binding features of trans-Atovaquone with cytochrome bc1 are shown in Figure 4. It shows two hydrogen bonded interactions with the protein residues of cytochrome bc1. The ketonic group of the drug molecule forms a hydrogen bond with the "NH" of Histidine (His-181) in one side and on the other side, the chlorine atom of 


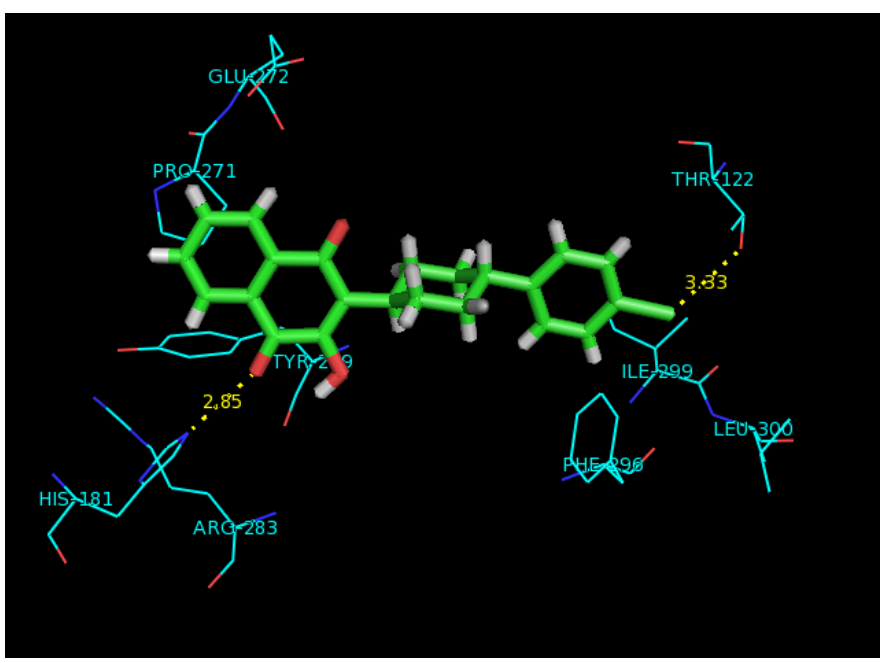

Figure 4. Energy minimized binding pattern of trans-Atovaquone at the active site of cytochrome bc1 of yeast.

Atovaquone forms a weak hydrogen bond with the "OH” group of Threonine (Thr-122) (Figure 4). The napthaquinone ring having three hydrophilic polar groups (two ketonic and one hydroxyl group) goes in to a polar cavity surrounded by residues Tryptophan (Tyr-279) with its $\mathrm{OH}$ directing to the cavity, Arginine (Arg-283) with its $\mathrm{NH}$ and Proline (Pro-271) with its NH pointing towards the cavity. The hydrophobic chloro benzene moiety of Atovaquone is surrounded by the hydrophobic group $\mathrm{CH}_{3}$ of Isoleucine (Ile-299), the alkyl group of Leucine (Leu-300) and $\pi$ cloud of the phenyl ring in Phenylalanine (Phe-296). There is an aromatic group near the chloro benzene group of Atovaquone but they are not close enough to form $\pi \ldots \pi$ type interactions, which could have further stabilized the interactions.

It is of interest to note that the model proposed earlier [20] based on docking studies with respect to similarities of Atovaquone with Stigmatellin suggested the participation of Glutamic acid (Glu-272) by invoking the presence of water, generating a hydrogen bonded motif. However, we do not find any such interaction involving Glutamic acid. This may be due to strong interaction with the Histidine (His-181) and the presence of O-H...Cl hydrogen bond with Threonine (Thr-122), the Glutamic acid residue is moved out of the reaction cavity.

\subsection{Binding Features of Cis-Atovaquone}

Unlike trans-Atovaquone which is a drug, the keto group of cis-Atovaquone (Figure 5) forms H-bond with the Glutamic acid (Glu272) of the cyto-bc1. This is due to the stearic repulsion of the chlorobenzene group which is perpendicular to the cyclohexyl ring of Atovaquone with the bulky hydrophobic groups like phenyl group of (Phe-296) $\mathrm{CH}_{3}$ groups of Leucine (Leu-300) and alkyl group of Isoleucine (Ile-299) of cyto-bc1. As a result, this keto group of Atovaquone goes away from the Histidine residue (the $\mathrm{C}=\mathrm{O}$ to $\mathrm{NH}$ of Histidine distance is $5.3 \AA$ in comparison to $2.85 \AA$ for the trans-isomer). Instead the second keto group (opposite to $\mathrm{OH}$ ) forms a hydrogen bond with the NH of Proline (Pro-271).

\subsection{Comparison of Binding Pattern of Trans- and Cis-Atovaquone}

It is seen from the overlapping pictures (Figure 6) of cis and trans-isomers of Atovaquone that major H-bonding interactions of cis-isomer arise out of its interaction with Glu-272 of cytbc1.The presence of Cl-H bonding of trans isomer imparts extra stability in addition to main H-bonding interaction with His-181. Both cis and transisomers are stabilized within the $Q$ pocket by different hydrophobic interactions. Thus, thermodynamic stability of the inhibitor within the $Q_{0}$ site of cytbc1 may not be the criteria of resisting electron transfer from ubiquinol to $2 \mathrm{Fe}-2 \mathrm{~S}$ centre of Rieske protein of cyt-bc1 complex by complicated bi-furcated path.

According to the recent mechanism proposed by Hunte et al. [18] and Barragan et al. [28], oxidation of quinol molecule at $Q_{0}$ site involves transfer of protons across the membrane and two electrons in a bifurcated path, one transfer from quinol to 2Fe-2S centre via a H-bond from Histidine. Trans-Atovaquone forms strong H-bond at 


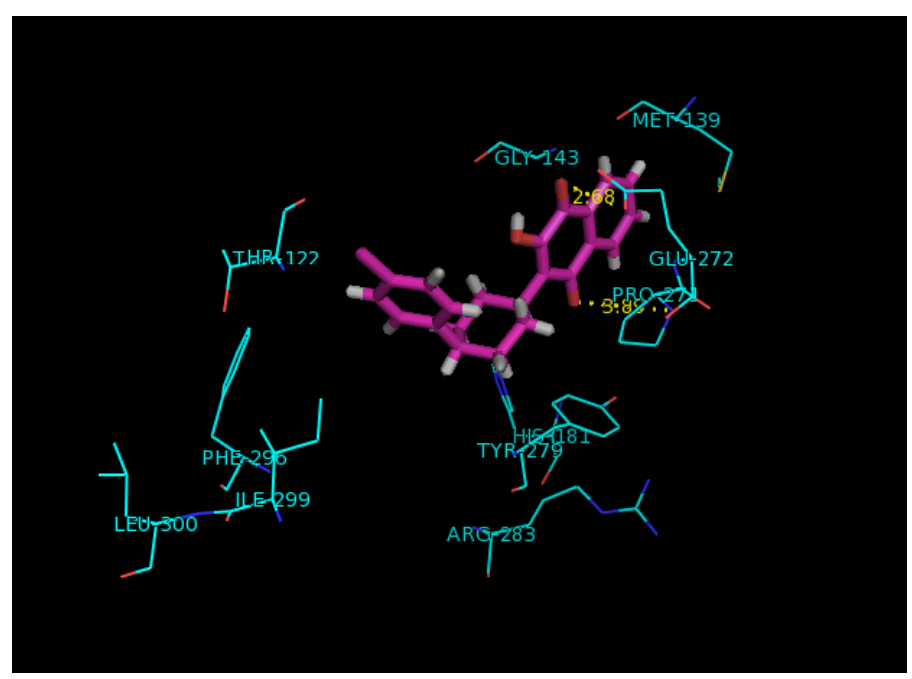

Figure 5. Energy minimized binding pattern of cis-Atovaquone at the active site of cytochrome bc1 of yeast.

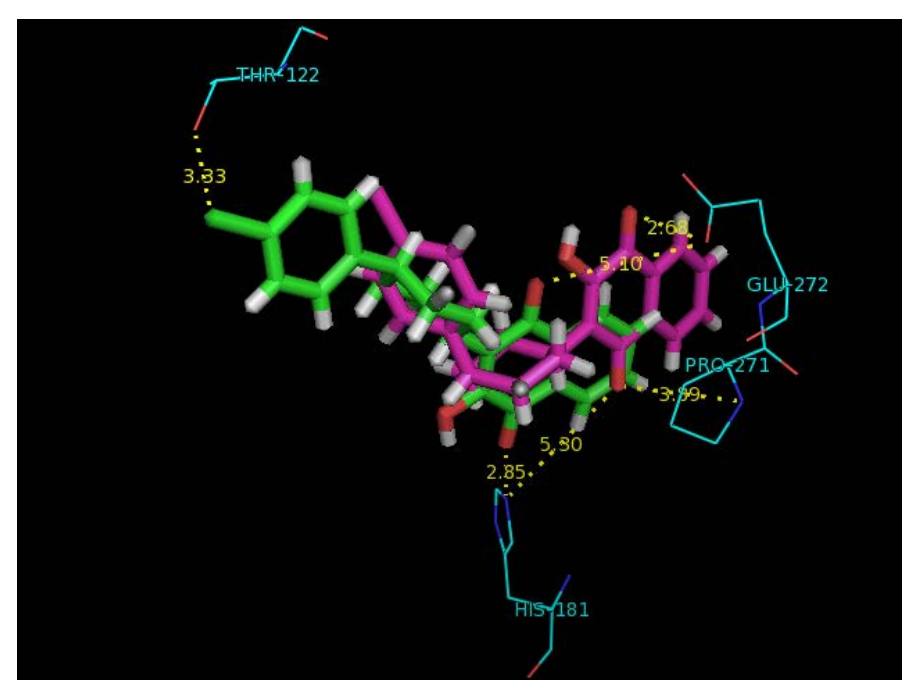

Figure 6. Comparison of energy minimized binding patterns of transAtovaquone and its cis-isomer at the active site of cytochrome bc1 of yeast.

this centre, hence block the electron transfer path through this route. This H-bonding interaction is absent with the cis-isomer and hence it fails to resist the electron transfer required for respiration of the parasites. This is possibly a major factor causing poor drug potency of cis-Atovaquone.

\section{Conclusion}

In the present research, we have presented for the first time the energy minimized binding pattern of cis-Atovaquone (which is not an anti-malaria drug) with cytochrome bc1of yeast using simple molecular docking calculation and compared with similar binding pattern of the drug molecule, trans-Atovaquone. We have hypothesized H-bonding interaction between $-\mathrm{NH}$ of His181 of Rieske protein and $\mathrm{C}=\mathrm{O}$ of Atovaquone plays a crucial role in dictating drug potency of Atovaquone.

\section{Acknowledgements}

SBM is thankful to Dr. Sekhar of IISc, Bangalore for his valuable suggestions and guidance. 


\section{References}

[1] WHO World Malaria Report 2014.

http://www.who.int/malaria/media/world malaria report 2014/en/

[2] Tuteja, R. (2007) Malaria-An Overview. FEBS Journal, 274, 4670-4679. http://dx.doi.org/10.1111/j.1742-4658.2007.05997.x

[3] Wongsrichanalai, C., Pickard, A.L., Wernsdorfer, W.H. and Meshnick, S.R. (2002) Epidemiology of Drug-Resistant Malaria. The Lancet Infectious Diseases, 2, 209-218. http://dx.doi.org/10.1016/S1473-3099(02)00239-6

[4] Joy, D., Feng, X., Mu, J., Furuya, T., Chotivanich, K., Krettl, A.U., Ho, M., Wang, A., White, N.J., Suh, E., Beerli, P. and Su, X. (2003) Early Origin and Recent Expansion of Plasmodium falciparum. Science, 300, 318-321. http://dx.doi.org/10.1126/science.1081449

[5] Dorn, A., Vippagunta, S.R., Matile, H., Jaquet, C., Vennerstrom, J.L. and Ridley, R.G. (1998) An Assessment of Drug-Haematin Binding as a Mechanism for Inhibition of Haematin Polymerisation by Quinoline Antimalarials. Biochemical Pharmacology, 55, 727-736. http://dx.doi.org/10.1016/S0006-2952(97)00510-8

[6] Banerjee, R., Liu, J., Beatty, W., Pelosof, L., Klemba, M. and Goldberg, D.E. (2002) Four Plasmepsins Are Active in the Plasmodium falciparum Food Vacuole, Including a Protease with an Active-Site Histidine. Proceedings of the National Academy of Sciences of the United States of America, 99, 990-995. http://dx.doi.org/10.1073/pnas.022630099

[7] Egan, T.J., Ross, D.C. and Adams, P.A. (1994) Quinoline Anti-Malarial Drugs Inhibit Spontaneous Formation of $\beta$-Haematin (Malaria Pigment). FEBS Letters, 352, 54-57. http://dx.doi.org/10.1016/0014-5793(94)00921-X

[8] Slater, A.F.G. and Cerami, A. (1992) Inhibition by Chloroquine of a Novel Haem Polymerase Enzyme Activity in Malaria Trophozoites. Nature, 355, 167-169. http://dx.doi.org/10.1038/355167a0

[9] Pandey, A.V., Babbarwal, V.K., Okoyeh, J.N., Joshi, R.M., Puri, S.K., Singh, R.L. and Chauhan, V.S. (2003) Hemozoin Formation in Malaria: A Two-Step Process Involving Histidine-Rich Proteins and Lipids. BBRC, 308, 736-743. http://dx.doi.org/10.1016/S0006-291X(03)01465-7

[10] Meshnick, S.R. (2002) Artemisinin: Mechanisms of Action, Resistance and Toxicity. International Journal for Parasitology, 13, 1655-1660. http://dx.doi.org/10.1016/S0020-7519(02)00194-7

[11] Mongan, P.D., Capacchione, J., Karaian, J., Dubois, D., Keneally, R. and Sharma, P. (2002) Pyruvate Improves Redox Status and Decreases Indicators of Hepatic Apoptosis during Hemorrhagic Shock in Swine. American Journal of Physiology-Heart and Circulatory Physiology, 283, 1634-1644. http://dx.doi.org/10.1152/ajpheart.01073.2001

[12] Basset, G.J.C., Quinlivan, E.P., Gregory, J.F. and Hanson, A.D. (2005) Folate Synthesis and Metabolism in Plants and Prospects for Biofortification. Crop Science, 45, 449-453. http://dx.doi.org/10.2135/cropsci2005.0449

[13] Myllykallio, H., Leduc, D., Filee, J. and Liebl, U. (2003) Life without Dihydrofolate Reductase FolA. Trends in Microbiology, 11, 220-223. http://dx.doi.org/10.1016/S0966-842X(03)00101-X

[14] Ridley, R.G. (2002) Medical Need, Scientific Opportunity and the Drive for Antimalarial Drugs. Nature, 415, 686-693. http://dx.doi.org/10.1038/415686a

[15] Fontaine, E., Ichas, F.O. and Bernardi, P.A. (1998) Ubiquinone-Binding Site Regulates the Mitochondrial Permeability Transition Pore. Journal of Biological Chemistry, 273, 25734-25740. http://dx.doi.org/10.1074/jbc.273.40.25734

[16] Tielens, A.G.M. and Hellemond, J.J.V. (1998) The Electron Transport Chain in Anaerobically Functioning Eukaryotes. BBA Bioenergetics, 1365, 71-78. http://dx.doi.org/10.1016/S0005-2728(98)00045-0

[17] Kroger, A. and Gwith, M.K. (1973) The Kinetics of the Redox Reactions of Ubiquinone Related to the Electron-Transport Activity in the Respiratory Chain. European Journal of Biochemistry, 34, 358-368. http://dx.doi.org/10.1111/j.1432-1033.1973.tb02767.x

[18] Hunte, C., Birth, D. and Kao, W.C. (2014) Structural Analysis of Atovaquone Inhibited Cytochrome bc1 Complex Revels the Molecular Basis of Antimalarial Drug Action. Nature Communication, 5029, 1-11. http://dx.doi.org/10.1038/ncomms5029

[19] Kessl, J.J., Lange, B.B., Merbitz, Z.T., Zwicker, K., Hill, P., Meunier, B., Hildur, P., lsdo, T., Hunte, C., Meshnick, S. and Trumpower, B.L. (2003) Molecular Basis for Atovaquone Binding to the Cytochrome bc1 Complex. Journal of Biological Chemistry, 278, 31312-31318. http://dx.doi.org/10.1074/jbc.M304042200

[20] Zhang, Z., Huang, L., Shulmeister, V.M., Chi, Y., Kim, K.K., Hung, L., Croftsk, A.R., Berry, E.A. and Kim, S. (1998) Electron Transfer by Domain Movement in Cytochrome bc1. Nature, 392, 677-684. http://dx.doi.org/10.1038/33612

[21] Nayek, S.K., Basumallick, S., Kanaujia, S.P., Sekhar, K., Ranganathan, K.R., Ananthalakshmi, V., Jeyaraman, G., Saralaya, S., Nagarajan, K. and Guru Row, T.N. (2013) Crystal Structures and Binding Studies of Atovaquone and Its Derivatives with Cytochrome bc1: A Molecular Basis for Drug Design. Crystal Engineering Communication, 15, 4871-4884. 
[22] Kirkpatrick, S., Gelatt, C.D. and Vecchi, M.P. (1983) Optimization by Simulated Annealing. Science, 220, 671-680. http://dx.doi.org/10.1126/science.220.4598.671

[23] Metropolis, N. and Ulam, S. (1949) The Monte Carlo Method. Journal of the American Statistical Association, 44, 335-341. http://dx.doi.org/10.1080/01621459.1949.10483310

[24] Meng, E.C., Shoichet, B.K. and Kuntz, I.D. (1992) Automated Docking with Grid-Based Energy Evaluation. Journal of Computational Chemistry, 13, 505-524. http://dx.doi.org/10.1002/jcc.540130412

[25] Solis, F.J. and Wets, R.J.B. (1981) Minimization by Random Search Techniques. Mathematics of Operations Research, 6, 19-30. http://dx.doi.org/10.1287/moor.6.1.19

[26] Boschitsch, A.H. and Fenley, M.O. (2004) Hybrid Boundary Element and Finite Difference Method for Solving the Nonlinear Poisson-Boltzmann Equation. Journal of Computational Chemistry, 25, 935-955. http://dx.doi.org/10.1002/jcc.20000

[27] Morris, G.M., Goodsell, D.S., Huey, R., Hart, W.E., Halliday, R.S., Belew, R.K. and Olson, A.J. (2001) Autodock Version 3.0.5. Scripps Research Institute, San Diego.

[28] Barragan, A.M., Crofts, A.R., Schulten, K and Solovyov, I.A. (2015) Identification of Ubiquinol Binding Motifs at the $\mathrm{Q}_{0}$ - Site of the Cytochrome bc1 Complex. Journal of Physical Chemistry B, 119, 433-447. http://dx.doi.org/10.1021/jp510022w 